\title{
RELIABILITY ASSESSMENT OF A SERIES SYSTEM WITH REDUNDANCY AND REPAIR FACILITIES
}

\author{
Yuriy Zhernovyi ${ }^{1}$, Bohdan Kopytko ${ }^{2}$ \\ ${ }^{I}$ Ivan Franko National University of Lviv, Lviv, Ukraine \\ ${ }^{2}$ Department of Mathematics, Czestochowa University of Technology \\ Czestochowa, Poland \\ yuriy.zhernovyy@lnu.edu.ua,bohdan.kopytko@im.pcz.pl
}

Received: 10 June 2020; Accepted: 27 August 2020

\begin{abstract}
In this paper, we propose a method for studying the reliability of series systems with redundancy and repair facilities. We consider arbitrary distributions of the units' time to failure and exponentially distributed recovery times. The approach based on the use of fictitious phases and hyperexponential approximations of arbitrary distributions by the method of moments. We consider cases of fictitious hyperexponential distributions with paradoxical and complex parameters. We define conditions for the variation coefficients of the gamma distributions and Weibull distributions, for which the best and same accuracy of calculating the steady-state probabilities is achieved in comparison with the results of simulation modeling.
\end{abstract}

MSC 2010: 60G10,60J28,60K25,93B40

Keywords: reliability, recoverable system, redundancy, series system, hyperexponential approximation, fictitious phases, method of moments

\section{Introduction}

Series systems of identical independent units with a common group of redundant units are common in engineering practice. Formally, such a structure appears if a system consists of units of several types [1]. A set of units of the same type can be considered as a "series system", for which there is a stock of spare units. It is reasonable to consider these spare units as unloaded; these units wait for being switched into operating position after one of the operating units has failed. Failed units are directed to a repair shop from where, after recovery, they again enter the system's stock. The switching of a spare unit into an operating position is usually assumed instantaneous.

Recoverable series systems differ by their recovery processes. Assume that although a system stops functioning in normal mode, but during recovering a currently failed unit new failures may appear. In principle, in this case one can observe 
even a situation when all system units have failed. It can happen if, for instance, a recovery process is very slow.

Consider a system that consists of $r=m+n$ identical units, namely, $n$ main operating units and $m$ unloaded redundant units. The system stops functioning in normal mode at the moment when the number of failed units reaches $m+1$. Assume that those $n-1$ units that were serviceable at the moment of system failure, in idle time during recovery, continue to operate and may fail. In addition, the number $c$ of repair facilities can be restricted $(c \leq r)$, so failed units can form a queue for recovering.

If we consider the described system as a queueing system, then in the absence of redundant units $(m=0)$, it is a classical closed queueing system [2]. The closed system is also known as a system with a finite number of sources or the Engset system.

In most academic approaches, a random time to failure and a random recovery time are assumed exponentially distributed for all units that give a possibility to use the Markov model for a reliability study. In this paper, we consider arbitrary distributions of the units' time to failure and exponentially distributed recovery time.

The method of potentials was used in [3] to construct an algorithm that makes it possible to calculate the steady-state distribution of the number of failed units for a single-channel closed system with an exponential distribution of the units' time to failure and an arbitrary distribution of recovery time. This method is not suitable for arbitrary distributions of the units' time to failure.

A method for calculating steady-state probability distributions of the multichannel closed queueing systems with exponential distribution of the time to failure and an arbitrary distribution of recovery time is proposed in [4].

Works [2, 4-8] show that the use of hyperexponential approximation (denoted by $H_{l}$ ) makes it possible to determine with high accuracy the steady-state probabilities of non-Markovian queuing systems. These probabilities are determined using solutions of a system of linear algebraic equations obtained by the method of fictitious phases. To find parameters of the $H_{l}$-approximation of a certain distribution, it is sufficient to solve the system of equations of the moments method. For the values $V<1$ of the variation coefficient, the roots of this system are complex-valued or paradoxical (i.e., negative or with probabilities that exceed the boundaries of the interval $[0,1]$ ) but in most cases as a result of summation of probabilities of microstates, their complex-valued and paradoxical parts are annihilated.

The purpose of the paper is to use the hyperexponential approximation method for studying the reliability of series systems with redundancy and repair facilities for the case of arbitrary distributions of the units' time to failure and exponentially distributed recovery time. We define conditions for the variation coefficients of the gamma distributions and Weibull distributions, for which the best or same accuracy of calculating the steady-state probabilities is achieved in comparison with the results of simulation modeling. 
Let $p_{k}$ be the steady-state probability of having $k$ failed units, then the stationary reliability indices of the system are determined by the formulas:

$$
K=\sum_{k=0}^{m} p_{k}, \quad N=\sum_{k=1}^{r} k p_{k} .
$$

Here $K$ is the stationary availability coefficient, and $N$ is the average number of failed units.

\section{Equations for steady-state probabilities}

The hyperexponential distribution of order $l$ is a phase-type distribution and provides for choosing one of $l$ alternative phases by a random process. With probability $y_{i}$, the process is at the $i$-th phase and is in it during an exponentially distributed time with a parameter $\theta_{i}$.

Suppose that the units' time to failure is distributed according to the hyperexponential law $H_{l}$ with probabilities $\alpha_{i}$ and parameters $\lambda_{i}(1 \leq i \leq l)$, and the units' recovery times are independent random variables distributed according to the exponential law with parameter $\mu$. Let us denote the corresponding closed queueing system by $H_{l} / M / c / r$ and we will use it for approximate calculation of the steadystate probabilities of the $G / M / c / r$ closed queueing system.

We will demonstrate the features of constructing equations for steady-state probabilities using the $H_{3} / M / c / r$ system as an example of the $H_{t} / M / c / r$ system.

Let us enumerate the $H_{3} / M / c / r$ system's states as follows: $x_{k(i, j, u)}$ is the state where there are $k$ failed units $(0 \leq k \leq r)$, and $i, j, u$ are the number of units for which the time to failure is in the first, second and third phase, respectively. We denote by $p_{k(i, j, u)}$ steady-state probabilities that the system is in the state $x_{k(i, j, u)}$. Since the process of changing the states of the system is Markovian with continuous time for the steady-state probabilities $p_{k(i, j, u)}$, we obtain a system of linear algebraic equations that follows from the Kolmogorov system of differential equations.

We use the notation:

$$
A_{k}=\left\{\begin{array}{ll}
k, & 1 \leq k \leq c-1, \\
c, & c \leq k \leq r-1 ;
\end{array} \quad B_{k}= \begin{cases}k+1, & 1 \leq k \leq c-1, \\
c, & c \leq k \leq r-1 .\end{cases}\right.
$$

Let us write the equations corresponding to the states $x_{0(n, 0,0)}, x_{0(0, n, 0)}$ and $x_{0(0,0, n)}$ :

$$
\begin{gathered}
-n \lambda_{1} p_{0(n, 0,0)}+\mu p_{1(n, 0,0)}=0, \quad-n \lambda_{2} p_{0(0, n, 0)}+\mu p_{1(0, n, 0)}=0, \\
-n \lambda_{3} p_{0(0,0, n)}+\mu p_{1(0,0, n)}=0 .
\end{gathered}
$$


On the example of the equation corresponding to the state $x_{0(i, n-i, 0)}$, we show the structure of the equations for the states $x_{0(i, n-i, 0)}, x_{0(i, 0, n-i)}$ and $x_{0(0, i, n-i)}$ :

$$
-\left(i \lambda_{1}+(n-i) \lambda_{2}\right) p_{0(i, n-i, 0)}+\mu p_{1(i, n-i, 0)}=0, \quad 1 \leq i \leq n-1 .
$$

Let us write the equation corresponding to the state $x_{0(i, j, n-i-j)}$ :

$$
\begin{array}{r}
-\left(i \lambda_{1}+j \lambda_{2}+(n-i-j) \lambda_{3}\right) p_{0(i, j, n-i-j)}+\mu p_{1(i, j, n-i-j)}=0, \\
1 \leq i \leq n-2,1 \leq j \leq n-1-i .
\end{array}
$$

Let us give examples of equations corresponding to the states $x_{k(n, 0,0)}, x_{k(0, n, 0)}$, $x_{k(0,0, n)}$ for $k \in\{1,2, \ldots, m\}$, and $x_{k(r-k, 0,0)}, x_{k(0, r-k, 0)}, x_{k(0,0, r-k)}$ for $k \in\{m+1, m+2, \ldots, r-1\}$ :

$$
\begin{aligned}
-\left(n \lambda_{1}+A_{k} \mu\right) p_{k(n, 0,0)} & +\alpha_{1}\left(n \lambda_{1} p_{k-1(n, 0,0)}+\lambda_{2} p_{k-1(n-1,1,0)}+\lambda_{3} p_{k-1(n-1,0,1)}\right)+ \\
& +B_{k} \mu p_{k+1(n, 0,0)}=0, \quad 1 \leq k \leq m-1 ; \\
-\left(n \lambda_{1}+A_{k} \mu\right) p_{m(n, 0,0)} & +\alpha_{1}\left(n \lambda_{1} p_{m-1(n, 0,0)}+\lambda_{2} p_{m-1(n-1,1,0)}+\lambda_{3} p_{m-1(n-1,0,1)}\right)+ \\
& +\alpha_{1} B_{k} \mu p_{m+1(n-1,0,0)}=0 ; \\
-\left((r-k) \lambda_{1}+A_{k} \mu\right) p_{k(r-k, 0,0)}+(r-k+1) \lambda_{1} p_{k-1(r-k+1,0,0)}+\lambda_{2} p_{k-1(r-k, 1,0)}+ & + \\
+ & \lambda_{3} p_{k-1(r-k, 0,1)}+\alpha_{1} B_{k} \mu p_{k+1(r-k-1,0,0)}=0, \quad m+1 \leq k \leq r-1 .
\end{aligned}
$$

By a similar principle, we compose the equations corresponding to the states $x_{k(i, n-i, 0)}, x_{k(i, 0, n-i)}, x_{k(0, i, n-i)}$ for $k \in\{1,2, \ldots, m\}$, and $x_{k(i, r-k-i, 0)}, x_{k(i, 0, r-k-i)}, x_{k(0, i, r-k-i)}$ for $k \in\{m+1, m+2, \ldots, r-2\}$ :

$$
\begin{aligned}
& -\left(i \lambda_{1}+(n-i) \lambda_{2}+A_{k} \mu\right) p_{k(i, n-i, 0)}+\alpha_{1}\left(i \lambda_{1} p_{k-1(i, n-i, 0)}+\right. \\
& \left.\quad+(n+1-i) \lambda_{2} p_{k-1(i-1, n+1-i, 0)}+\lambda_{3} p_{k-1(i-1, n-i, 1)}\right)+ \\
& \quad+\alpha_{2}\left((i+1) \lambda_{1} p_{k-1(i+1, n-1-i, 0)}+(n-i) \lambda_{2} p_{k-1(i, n-i, 0)}+\right. \\
& \left.+\lambda_{3} p_{k-1(i, n-1-i, 1)}\right)+B_{k} \mu p_{k+1(i, n-i, 0)}=0,1 \leq k \leq m-1,1 \leq i \leq n-1 ; \\
& -\left(i \lambda_{1}+(n-i) \lambda_{2}+A_{k} \mu\right) p_{m(i, n-i, 0)}+\alpha_{1}\left(i \lambda_{1} p_{m-1(i, n-i, 0)}+\right. \\
& \left.\quad+(n+1-i) \lambda_{2} p_{m-1(i-1, n+1-i, 0)}+\lambda_{3} p_{m-1(i-1, n-i, 1)}\right)+ \\
& \quad+\alpha_{2}\left((i+1) \lambda_{1} p_{m-1(i+1, n-1-i, 0)}+(n-i) \lambda_{2} p_{m-1(i, n-i, 0)}+\right. \\
& \left.+\lambda_{3} p_{m-1(i, n-1-i, 1)}\right)+B_{k} \mu\left(\alpha_{1} p_{m+1(i-1, n-i, 0)}+\alpha_{2} p_{m+1(i, n-1-i, 0)}\right)=0,1 \leq i \leq n-1 ; \\
& -\left(i \lambda_{1}+(r-k-i) \lambda_{2}+A_{k} \mu\right) p_{k(i, r-k-i, 0)}+(i+1) \lambda_{1} p_{k-1(i+1, r-k-i, 0)}+ \\
& \quad+(r-k-i+1) \lambda_{2} p_{k-1(i, r-k-i+1,0)}+\lambda_{3} p_{k-1(i, r-k-i, 1)}+ \\
& +B_{k} \mu\left(\alpha_{1} p_{k+1(i-1, r-k-i, 0)}+\alpha_{2} p_{k+1(i, r-k-i-1,0)}\right)=0, m+1 \leq k \leq r-2,1 \leq i \leq r-k-1 .
\end{aligned}
$$


Let us write the equations corresponding to the states $x_{k(i, j, n-i-j)}$ for $k \in\{1,2, \ldots, m\}$, and $x_{k(i, j, r-k-i-j)}$ for $k \in\{m+1, m+2, \ldots, r-3\}$ :

$$
\begin{aligned}
& -\left(i \lambda_{1}+j \lambda_{2}+(n-i-j) \lambda_{3}+A_{k} \mu\right) p_{k(i, j, n-i-j)}+\alpha_{1}\left(i \lambda_{1} p_{k-1(i, j, n-i-j)}+\right. \\
& \left.+(j+1) \lambda_{2} p_{k-1(i-1, j+1, n-i-j)}+(n+1-i-j) \lambda_{3} p_{k-1(i-1, j, n+1-i-j)}\right)+ \\
& +\alpha_{2}\left((i+1) \lambda_{1} p_{k-1(i+1, j-1, n-i-j)}+j \lambda_{2} p_{k-1(i, j, n-i-j)}+\right. \\
& \left.+(n+1-i-j) \lambda_{3} p_{k-1(i, j-1, n+1-i-j)}\right)+\alpha_{3}\left((i+1) \lambda_{1} p_{k-1(i+1, j, n-1-i-j)}+\right. \\
& \left.+(j+1) \lambda_{2} p_{k-1(i, j+1, n-1-i-j)}+(n-i-j) \lambda_{3} p_{k-1(i, j, n-i-j)}\right)+ \\
& +B_{k} \mu p_{k+1(i, j, n-i-j)}=0,1 \leq k \leq m-1,1 \leq i \leq n-2,1 \leq j \leq n-i-1 \text {; } \\
& -\left(i \lambda_{1}+j \lambda_{2}+(n-i-j) \lambda_{3}+A_{k} \mu\right) p_{m(i, j, n-i-j)}+\alpha_{1}\left(i \lambda_{1} p_{m-1(i, j, n-i-j)}+\right. \\
& \left.+(j+1) \lambda_{2} p_{m-1(i-1, j+1, n-i-j)}+(n+1-i-j) \lambda_{3} p_{m-1(i-1, j, n+1-i-j)}\right)+ \\
& +\alpha_{2}\left((i+1) \lambda_{1} p_{m-1(i+1, j-1, n-i-j)}+j \lambda_{2} p_{m-1(i, j, n-i-j)}+\right. \\
& \left.+(n+1-i-j) \lambda_{3} p_{m-1(i, j-1, n+1-i-j)}\right)+\alpha_{3}\left((i+1) \lambda_{1} p_{m-1(i+1, j, n-1-i-j)}+\right. \\
& \left.+(j+1) \lambda_{2} p_{m-1(i, j+1, n-1-i-j)}+(n-i-j) \lambda_{3} p_{m-1(i, j, n-i-j)}\right)+ \\
& +B_{k} \mu\left(\alpha_{1} p_{m+1(i-1, j, n-i-j)}+\alpha_{2} p_{m+1(i, j-1, n-i-j)}+\alpha_{3} p_{m+1(i, j, n-1-i-j)}\right)=0, \\
& 1 \leq i \leq n-2,1 \leq j \leq n-i-1 \text {; } \\
& -\left(i \lambda_{1}+j \lambda_{2}+(r-k-i-j) \lambda_{3}+A_{k} \mu\right) p_{k(i, j, r-k-i-j)}+ \\
& +(i+1) \lambda_{1} p_{k-1(i+1, j, r-k-i-j)}+(j+1) \lambda_{2} p_{k-1(i, j+1, r-k-i-j)}+ \\
& +(r-k-i-j+1) \lambda_{3} p_{k-1(i, j, r-k-i-j+1)}+ \\
& +B_{k} \mu\left(\alpha_{1} p_{k+1(i-1, j, r-k-i-j)}+\alpha_{2} p_{k+1(i, j-1, r-k-i-j)}+\alpha_{3} p_{k+1(i, j, r-k-i-j-1)}\right)=0, \\
& m+1 \leq k \leq n-3,1 \leq i \leq r-k-2,1 \leq j \leq r-k-i-1 .
\end{aligned}
$$

Let us write the equation corresponding to the state $x_{r(0,0,0)}$ :

$$
-c \mu p_{r(0,0,0)}+\lambda_{1} p_{r-1(1,0,0)}+\lambda_{2} p_{r-1(0,1,0)}+\lambda_{3} p_{r-1(0,0,1)}=0
$$

The normalization condition closes the system of equations for steady-state probabilities:

$$
\sum_{k=0}^{m} \sum_{i=0}^{n} \sum_{j=0}^{n-i} p_{k(i, j, n-i-j)}+\sum_{k=m+1}^{r} \sum_{i=0}^{r-k} \sum_{j=0}^{r-k-i} p_{k(i, j, r-k-i-j)}=1 .
$$


Solving the system obtained by the method of fictitious phases, we find the steady-state probabilities $p_{k}$ of having $k$ failed units, using the formulas

$$
p_{k}=\sum_{i=0}^{n} \sum_{j=0}^{n-i} p_{k(i, j, n-i-j)}, 0 \leq k \leq m ; \quad p_{k}=\sum_{i=0}^{r-k} \sum_{j=0}^{r-k-i} p_{k(i, j, r-k-i-j)}, m+1 \leq k \leq r .
$$

If we consider, for example, the $H_{5} / M / c / r$ system, then equalities (11) have the form

$$
\begin{aligned}
& p_{k}=\sum_{i=0}^{n} \sum_{j=0}^{n-i} \sum_{s=0}^{n-i-j} \sum_{u=0}^{n-i-j-s} p_{k(i, j, s, u, n-i-j-s-u)}, 0 \leq k \leq m ; \\
& p_{k}=\sum_{i=0}^{r-k} \sum_{j=0}^{r-k-i} \sum_{s=0}^{r-k-i-j} \sum_{u=0}^{r-k-i-j-s} p_{k(i, j, s, u, r-k-i-j-s-u)}, \quad m+1 \leq k \leq r .
\end{aligned}
$$

\section{Numerical results}

In this section, we present the results of using the $H_{5} / M / c / r$ and $H_{6} / M / c / r$ systems to calculate the probabilities $p_{k}$ for the $G / M / c / r$ series recoverable systems with redundancy. We consider the gamma distributions and Weibull distributions of units' time to failure and exponentially distributed units' recovery time.

Let $\Gamma(V)$ and $W(V)$ denote the gamma distribution and Weibull distribution with coefficients of variation $V$.

We determine the values of the variation coefficients of the $\Gamma(V)$ and $W(V)$ distributions, for which the conditions

$$
\Delta_{(6,5)}<2 \cdot 10^{-3}
$$

or

$$
2 \cdot 10^{-3} \leq \Delta_{(6,5)}<2 \cdot 10^{-2}
$$

hold. Here $\Delta_{(6,5)}=\sum_{k=0}^{r}\left|p_{k(6)}-p_{k(5)}\right|$ gives an opportunity to estimate the deviation of distributions $\left\{p_{k(6)}\right\}$ from distributions $\left\{p_{k(5)}\right\}$, where $p_{k(l)}$ are values of probabilities $p_{k}$ obtained using the $H_{l} / M / c / r$ system as an approximation of the $G / M / c / r$ series recoverable system. If the condition (13) is fulfilled, then the distribution $\left\{p_{k(6)}\right\}$ is a more accurate approximation to the true steady-state distribution $\left\{p_{k}\right\}$ than the distribution $\left\{p_{k(s i m)}\right\}$, obtained using the GPSS World simulation system $[9,10]$. If $\Delta_{(6,5)} \in\left[2 \cdot 10^{-3}, 2 \cdot 10^{-2}\right)$, then the same accuracy of calculating the 
steady-state probabilities $\left\{p_{k}\right\}$ is achieved as for the results of simulation modeling. We arrived at these conclusions after comparing the distributions $\left\{p_{k}\right\}$ and $\left\{p_{k(\text { sim })}\right\}$ for the $M / M / c / r$ series recoverable systems. The mathematical description of such systems is given by a "birth-death process". The GPSS World simulation model of the series recoverable system with redundancy is constructed in [10].

Let us take $n=4, m=3, c \in\{1,2, \ldots, 5\}, T=4$ and $\mu=0.5$, where $T$ is the mean units' time to failure. In the case of absence of redundant units $(m=0)$, the distribution $\left\{p_{k}\right\}$ is independent of the distribution of units' time to failure [11], therefore it is determined as the exact distribution for the $M / M / c / r$ closed system.

The values of the variation coefficients of the $\Gamma(V)$ and $W(V)$ distributions, for which the conditions (13) and (14) hold, are presented in Tables 1 and 2, respectively. Of all the intervals corresponding to the $\Gamma(V)$ distributions, the values $V=0.5$ and $V=1 / \sqrt{2}$ of the variation coefficient should be excluded, since for the $\Gamma(0.5)$ distribution it is not possible to construct approximations with the help of hyperexponential distributions of order higher than the third, and hyperexponential approximations do not exist for the $\Gamma(1 / \sqrt{2})$ distribution.

The results of calculation of reliability steady-state indices for different distributions of units' time to failure are given in Table 3.

Table 1 . List of the $G / M / c / 7$ series recoverable systems with the gamma distributions and Weibull distributions of units' time to failure for which condition

$$
\Delta_{(6,5)}<2 \cdot 10^{-3} \text { holds }
$$

\begin{tabular}{|c|c|c|}
\hline$c$ & $\begin{array}{c}\text { Values of } V \\
\text { for } \Gamma(V)\end{array}$ & $\begin{array}{c}\text { Values of } V \\
\text { for } W(V)\end{array}$ \\
\hline 1 & {$[0.001,2.8]$} & {$[0.001,2.2]$} \\
\hline 2 & {$[0.001,1.8]$} & {$[0.001,1.6]$} \\
\hline 3 & {$[0.2,1.6]$} & {$[0.2,1.5]$} \\
\hline 4 & {$[0.7,1.5]$} & {$[0.7,1.2]$} \\
\hline 5 & {$[0.7,1.5]$} & {$[0.7,1.2]$} \\
\hline
\end{tabular}

Table 2. List of the $G / M / c / 7$ series recoverable systems with the gamma distributions and Weibull distributions of units' time to failure for which condition $\Delta_{(6,5)} \in\left[2 \cdot 10^{-3}, 2 \cdot 10^{-2}\right)$ holds

\begin{tabular}{|c|c|c|}
\hline$c$ & Values of $V$ for $\Gamma(V)$ & Values of $V$ for $W(V)$ \\
\hline 1 & {$[2.9,25]$} & {$[2.3,10]$} \\
\hline 2 & {$[1.9,5.8]$} & {$[1.7,3.2]$} \\
\hline 3 & {$[0.001,0.1],[1.7,3.9]$} & {$[0.001,0.1],[1.6,2.6]$} \\
\hline 4 & {$[1.6,3.3]$} & {$[1.3,2.5]$} \\
\hline 5 & {$[1.6,3.3]$} & {$[1.3,2.6]$} \\
\hline
\end{tabular}


The data in Tables 1-3 show that a high accuracy of approximation is achieved for a wider range of values of the variation coefficient of the $\Gamma(V)$ distribution compared to the $W(V)$ distribution. Approximation accuracy decreases and the reliability indices improve with increasing the number $c$ of repair facilities. The reliability indices deteriorate with a decrease in the number $m$ of redundant units. An increase in the coefficients of variation of the $\Gamma(V)$ and $W(V)$ distributions leads to a deterioration of reliability indices.

Table 3. Results of the calculation of steady-state reliability indices for different distributions of units' time to failure

\begin{tabular}{|c|c|c|c|c|c|c|c|}
\hline $\begin{array}{l}\text { Distribution } \\
\text { name }\end{array}$ & $m$ & $\begin{array}{l}\text { Indice } \\
\text { name }\end{array}$ & $c=1$ & $c=2$ & $c=3$ & $c=4$ & $c=5$ \\
\hline \multirow{3}{*}{$\Gamma(0.001)$} & \multirow{3}{*}{3} & $\Delta_{(6,5)}$ & $3.23 \cdot 10^{-4}$ & $3.34 \cdot 10^{-3}$ & $1.01 \cdot 10^{-2}$ & - & - \\
\hline & & $N$ & 4.9240 & 2.8275 & 2.0832 & - & - \\
\hline & & $K$ & 0.1596 & 0.6702 & 0.8766 & - & - \\
\hline \multirow{3}{*}{$\Gamma(0.7)$} & \multirow{3}{*}{3} & $\Delta_{(6,5)}$ & $1.12 \cdot 10^{-7}$ & $3.91 \cdot 10^{-6}$ & $1.81 \cdot 10^{-6}$ & $1.01 \cdot 10^{-6}$ & $1.22 \cdot 10^{-6}$ \\
\hline & & $N$ & 4.9100 & 2.8769 & 2.1238 & 1.9630 & 1.9354 \\
\hline & & $K$ & 0.1647 & 0.6368 & 0.8340 & 0.8848 & 0.8920 \\
\hline \multirow{3}{*}{$W(0.7)$} & \multirow{3}{*}{3} & $\Delta_{(6,5)}$ & $1.51 \cdot 10^{-5}$ & $5.47 \cdot 10^{-5}$ & $1.02 \cdot 10^{-4}$ & $1.16 \cdot 10^{-4}$ & $1.12 \cdot 10^{-4}$ \\
\hline & & $N$ & 4.9095 & 2.8798 & 2.1260 & 1.9625 & 1.9341 \\
\hline & & $K$ & 0.1644 & 0.6344 & 0.8313 & 0.8828 & 0.8901 \\
\hline \multirow{3}{*}{$\Gamma(2)$} & \multirow{3}{*}{3} & $\Delta_{(6,5)}$ & $7.49 \cdot 10^{-4}$ & $2.96 \cdot 10^{-3}$ & $4.47 \cdot 10^{-3}$ & $5.61 \cdot 10^{-3}$ & $5.47 \cdot 10^{-3}$ \\
\hline & & $N$ & 4.8831 & 3.0314 & 2.2232 & 1.9438 & 1.8826 \\
\hline & & $K$ & 0.1551 & 0.5267 & 0.7178 & 0.7947 & 0.8087 \\
\hline \multirow{3}{*}{$W(2)$} & \multirow{3}{*}{3} & $\Delta_{(6,5)}$ & $1.39 \cdot 10^{-3}$ & $5.81 \cdot 10^{-3}$ & $7.92 \cdot 10^{-3}$ & $9.20 \cdot 10^{-3}$ & $9.04 \cdot 10^{-3}$ \\
\hline & & $N$ & 4.8869 & 2.9992 & 2.1999 & 1.9969 & 1.9353 \\
\hline & & $K$ & 0.1601 & 0.5553 & 0.7510 & 0.8118 & 0.8261 \\
\hline \multirow{3}{*}{$\Gamma(3)$} & \multirow{3}{*}{3} & $\Delta_{(6,5)}$ & $2.15 \cdot 10^{-3}$ & $7.73 \cdot 10^{-3}$ & $1.31 \cdot 10^{-2}$ & $1.62 \cdot 10^{-2}$ & $1.58 \cdot 10^{-2}$ \\
\hline & & $N$ & 4.8814 & 3.1156 & 2.2742 & 1.9308 & 1.8530 \\
\hline & & $K$ & 0.1419 & 0.4651 & 0.6518 & 0.7397 & 0.7562 \\
\hline \multirow{2}{*}{ All } & \multirow{2}{*}{0} & $N$ & 2.1905 & 1.4713 & 1.3443 & 1.3333 & - \\
\hline & & $K$ & 0.0952 & 0.1839 & 0.1967 & 0.1975 & - \\
\hline
\end{tabular}

\section{Conclusions}

This paper shows that the application of hyperexponential approximations of distributions of the units' time to failure allow us to calculate steady-state probabilities of the series recoverable system with redundancy and repair facilities. We find these probabilities using solutions of a system of linear algebraic equations obtained 
by the method of fictitious phases. To obtain parameters of $H_{l}$ - approximation of a certain distribution, it is necessary to solve the system of equations of the moments method. Computing the deviations $\Delta_{(6,5)}$ allows us to track the accuracy of approaching distributions $\left\{p_{k(l)}\right\}$ to the true distribution $\left\{p_{k}\right\}$. We defined conditions for the variation coefficients of the gamma distributions and Weibull distributions, for which the best or the same accuracy of calculating the steady-state probabilities is achieved compared with the case of using simulation models.

\section{References}

[1] Ushakov, I. (2012). Probabilistic Reliability Models. Hoboken: John Wiley \& Sons.

[2] Zhernovyi, Yu., \& Kopytko, B. (2020). Calculating steady-state probabilities of single-channel closed queueing systems using hyperexponential approximation. J. Appl. Math. Comput. Mech., 19(1), 113-120.

[3] Zhernovyi, Yu.V., \& Zhernovyi, K.Yu. (2015). Method of potentials for a closed system with queue length dependent service times. J. of Communications Technology and Electronics, 60(12), 1341-1347.

[4] Aliyev, S.A., Yeleyko, Y.I., \& Zhernovyi, Yu.V. (2019). Calculating steady-state probabilities of closed queueing systems using hyperexponential approximation. Caspian J. of Appl. Math., Economics and Ecology, 7(1), 46-55.

[5] Ryzhikov, Yu.I., \& Ulanov, A.V. (2016). Application of hyperexponential approximation in the problems of calculating non-Markovian queuing systems. Vestnik of Tomsk State University. Management, Computer Engineering and Informatics, 3(36), 60-65 (in Russian).

[6] Zhernovyi, Yu.V. (2018). Calculating steady-state characteristics of single-channel queuing systems using phase-type distributions. Cybernetics and Systems Analysis, 54(5), 824-832.

[7] Zhernovyi, Yu. \& Kopytko, B. (2019). Calculating steady-state probabilities of queueing systems using hyperexponential approximation. J. Appl. Math. Comput. Mech., 18(2), 111-122.

[8] Zhernovyi, Yu. (2019). Computing Non-Markovian Queues Using Hyperexponential Distributions. Riga: LAP Lambert Academic Publishing.

[9] Zhernovyi, Yu. (2015). Creating Models of Queueing Systems Using GPSS World. Saarbrücken: LAP Lambert Academic Publishing.

[10] Zhernovyi, Yu. V. (2020). Simulation Models of Reliability. Zhytomyr: PC "ZhytomyrPolygraph" (in Ukrainian).

[11] König, D., Rykov, V.V., \& Schmidt, F. (1981). Stationary queuing systems with dependencies. Itogi Nauki $i$ Tekhniki. Ser. Probability Theory, Math. Stat. Theor Cybernet. 18, 95-186 (in Russian). 